\title{
Constant Proportion Portfolio Insurance Strategies under Cumulative Prospect Theory with Reference Point Adaptation
}

\author{
Anil Khuman ${ }^{1}$, Nick Constantinou*2 ${ }^{* 2}$ and Steve Phelps ${ }^{1}$ \\ ${ }^{1}$ Centre for Computational Finance and Economic Agents (CCFEA), University of \\ Essex, Colchester CO4 3SQ, UK \\ ${ }^{2}$ Essex Business School (EBS), University of Essex, Colchester CO4 3SQ, UK
}

\begin{abstract}
Constant Proportion Portfolio Insurance (CPPI) is a significant and highly popular investment strategy within the structured product market. This has led to recent work which attempts to explain the popularity of CPPI by showing that it is compatible with Cumulative Prospect Theory (CPT). We demonstrate that this cannot explain the popularity of ratcheted CPPI products which lock-in gains during strong growth in the portfolio. In this paper we conjecture that CPPI investors not only follow CPT, but crucially that they also adapt their reference point over time. This important distinction explains investors preference for ratcheted products.
\end{abstract}

JEL: G11; G13.

Keywords: Constant Proportion Portfolio Insurance; Ratchets; Cumulative Prospect Theory; Adaptive Reference Point.

\section{Introduction}

In the current financial climate investors have a greater concern about risk. Naturally this impacts their choice of investment product as they have become more wary of losing money. Portfolio insurance (PI) offers a solution to this problem by giving investors a guarantee on the minimum payoff they will receive at maturity. Typically the guarantee is on the initial amount invested, providing investors with peace of mind that they will at the very least not lose their original capital.

There are several different approaches to providing portfolio insurance. A common approach is Constant Proportion Portfolio Insurance (CPPI) (Black and Jones, 1987; Perold and Sharpe, 1988). CPPI is a portfolio insurance strategy that dynamically manages capital between a risky and risk-free asset with the goal of providing a minimum guarantee at maturity. Although the strategy was developed many years ago, it is still very popular with investors, and together with its variants it accounts for a significant proportion of the structured fund market whose importance has come to the attention of regulators (Pain, 2008).

The original CPPI model is formulated in continuous time and assumes instantaneous trading and smooth price changes. However, in reality these assumptions are violated. This introduces the notion

\footnotetext{
${ }^{*}$ Corresponding author. Tel.: ++ 44 (0) 1206 873919, Fax: ++ 44 (0) 1206 873429, E-mail: nconst@essex.ac.uk.
} 
of gap risk - the risk that the portfolio value will not meet the guarantee at maturity. Discontinuities in the price of the risky asset, trading frictions and a lack of liquidity all contribute to gap risk. To this end Cont and Tankov (2009) examine the impact of price jumps using historical parameter estimates and find that although there is some gap risk, it is relatively low. Bertrand and Prigent (2002) apply extreme value theory to allow higher multiplier values when a quantile hedging approach is taken. Maringer and Ramtohul (2011) employ simulated data in the form of a GARCH risky asset price process, while Dichtl and Drobetz (2011) and Do (2002) use historical data from large indices. Do and Faff (2004) successfully apply CPPI to a futures market. Balder et al. (2009) investigate discrete trading models in the presence of transaction costs by using a calendar rebalancing approach. Alternative strategies whereby rebalancing is triggered by movements in the risky asset are investigated by Jessen (2010), Dichtl and Drobetz (2011), Do (2002) and Maringer and Ramtohul (2011).

The control parameter of the CPPI, the multiplier, governs the amount of exposure the portfolio has to the risky asset. It can therefore be considered a proxy for the risk aversion of the investor. Although values as a high as 17 have been recommended by Bertrand and Prigent (2002), typically multiplier values of between 2 and 5 are used (Hamidi et al., 2009; Jessen, 2010). Chen et al. (2008) and Ben Ameur (2010) use dynamic multiplier approaches, demonstrating their potential to outperform standard static multiplier models.

Although the basic CPPI assumes a floor that grows at a constant risk-free rate, the implementation of more complex floor dynamics are investigated by Boulier and Kanniganti (1995), Ben Ameur and Prigent (2011) and Lee et al. (2010). Boulier and Kanniganti (1995) find that the addition of a ratchet increases the performance of the CPPI in comparison to a leverage constrained implementation. The increasing of the floor conditional on increases in the portfolio value is commonly termed ratcheting. The concept is similar to the Time Invariant Portfolio Protection (TIPP) introduced by Estep and Kritzman (1988); Choie and Seff (1989). In TIPP an initial floor level is set equal to the guarantee which is below the initial portfolio value. The floor/guarantee value is then continuously revised upwards on gains in the portfolio value. Because the floor is not discounted back from maturity a constant risk-free rate is not required. A benefit of this is that the maturity of the investment does not have to be defined in advance. However, this means that the strategy is unable to ensure $100 \%$ of the initial portfolio value.

The popularity of CPPI investments can be explained by the preferences of investors under certain utility functions with some restrictions. It has been shown that the CPPI strategy is utility maximising for the piecewise Hyperbolic Absolute Risk Aversion (HARA) utility function. Although with the introduction of leverage and trading constraints this is no longer true (Black and Perold, 1992). When the guaranteed amount is considered as a subsistence level the CPPI is optimal under Constant Relative Risk Aversion (CRRA) (Basak, 2002; Branger et al., 2010). However, more recent research indicates that the desire for protected products, including the CPPI, is compatible with the Cumulative Prospect Theory (CPT) framework. Indeed Dichtl and Drobetz (2011), Dierkes et al. (2010) and Vrecko and Branger (2009) all provide evidence that a CPT investor generally favours portfolio insurance over other strategies, e.g. constant mix and buy-and-hold.

It is typically assumed that investors evaluate their utility based on a function of their terminal wealth. Alternatively, under prospect theory it is the difference between this terminal wealth and some fixed reference level. However, research suggests that investors' actual evaluation of investment choices is far more complex; Arkes et al. (2008) provide evidence that investors adapt their reference point in relation to changes in investment value. A much more rapid increase in the reference point is seen for gains than a decrease for losses. Lin et al. (2006) investigate the effect of regret on the perception of an investor's performance when giving the choice between three stocks. By framing other possible investments as missed opportunities they find that an investor's regret is "most influenced by what their outcomes might have been had they not invested, by their expected outcomes and by the best-performing unchosen stocks"،

This paper aims to explain investors' preference for ratcheted guarantee investment products by posit- 
ing that they use cumulative prospect theory with an adaptive reference point. We demonstrate that a dynamic reference point, increasing in relation to the maximum portfolio value, is crucial for an investor to select a ratcheted CPPI strategy over the standard unratcheted one.

The structure of this paper is as follows. In Section 2 we provide a detailed description of the CPPI strategies. Section 3 describes GJR-GARCH model used to simulate the risky asset price dynamics. In Section 4 we describe the cumulative prospect theory model. The results are presented and discussed in Section 5, and finally Section 6 concludes.

\section{Background}

\subsubsection{Standard Model}

As stated in the introduction, the canonical CPPI model, introduced by Black and Jones (1987); Perold and Sharpe (1988), makes a number of simplifications and assumptions that renders it unsuitable for practical implementation. Firstly, it is not possible to trade instantaneously and the risky asset does not exhibit continuous and smooth price changes. Secondly, the assumption of the risky asset following a geometric Brownian motion (GBM) is contrary to empirical evidence. Thirdly, shorting of the risky asset is often not possible and there is a finite limit on the amount of capital that may be borrowed. Finally, it is unlikely that the risk-free rate would remain constant during the entire investment period. Aside from this last point, all the other assumptions are relaxed in this paper. Keeping the risk-free rate constant is considered acceptable since it contributes a small amount of risk to the strategy in comparison to the risky asset. The following subsections presents the discrete-timed CPPI model used and its extensions.

\subsection{CPPI in Discrete Time}

In this subsection we present a discrete time CPPI model where the following convention is adopted: a horizon $T$ and $n+1$ equidistant points:

$0=t_{0}<t_{1} \ldots<t_{n-1}<t_{n}=T$, such that $t_{k+1}-t_{k}=\frac{T}{n}$ for $k=0, \ldots, n-1$. Where $n$ is the number of times the price of the risky asset is observed after the initial construction of the portfolio.

Every period the CPPI model rebalances capital between a risky asset $S$ and a risk-free asset that grows at the constant rate $r$. At any time $t_{k}$, a floor $F_{t_{k}}$ is calculated by discounting back from maturity T the guarantee amount $G_{t_{k}}=g_{t_{k}} V_{0}$ using the risk-free rate $r$, where $V_{0}$ is the initial capital and $g_{0}$ the percentage of that amount initially guaranteed i.e. $g_{0}=100 \%$ guarantees the initial investment. The cushion $C_{t_{k}}$ is defined as the difference between the portfolio value $V_{t_{k}}$ and the floor. The investment in the risky asset $E_{t}$, termed the exposure, is defined as a constant multiple $m$ of the cushion, whereby a higher $m$ results in a greater exposure. The remainder of the capital $B_{t_{k}}$ is invested in the risk-free asset. The above can be summarised in the following set of equations:

$$
\begin{aligned}
V_{t_{k}} & =F_{t_{k}}+C_{t_{k}} \\
F_{t_{k}} & =G_{t_{k}} e^{-r\left(T-t_{k}\right)} \\
G_{t_{k}} & =g_{t_{k}} V_{0} \\
E_{t_{k}} & =m C_{t_{k}} \\
B_{t_{k}} & =V_{t_{k}}-E_{t_{k}} .
\end{aligned}
$$

The time subscript on the guarantee percentage $g$ and the guaranteed amount $G$ is required for instances where the value of the guarantee and floor are conditional on some process. This is discussed later.

The progression of the CPPI is driven by changes in the risky asset price $S_{t_{k}}$. When $S_{t_{k}}$ increases at a rate greater than $r$, then more capital is invested in the risky asset by selling some of the risk-free asset. 
If $S_{t_{k}}$ grows at a rate less than $r$ then some of the risky asset is sold and the proceeds are invested in the risk-free asset. In both cases the amount bought or sold is such that Equation (1d) is always satisfied. Thus $V_{t_{k}}$ can be expressed in terms of $S_{t_{k}}$ as (see e.g. Balder et al. (2009) for derivation)

$$
V_{t_{k}}= \begin{cases}F_{t_{k}}+\left(V_{t_{k-1}}-F_{t_{k-1}}\right)\left(m \frac{S_{t_{k}}}{S_{t_{k-1}}}-(m-1) e^{r \frac{T}{n}}\right) & \text { if } \quad V_{t_{k-1}}>F_{t_{k-1}} \\ V_{t_{k-1}} e^{r \frac{T}{n}} & \text { if } \quad V_{t_{k-1}} \leq F_{t_{k-1}}\end{cases}
$$

Note that Equation (2) exhibits clear path dependence of the value of the CPPI in discrete time, provided the floor has not been hit. Also note that the definition is independent of the price process governing $S$. In the following subsection we describe some common extensions to the basic CPPI framework.

\subsection{Model Extensions}

\subsubsection{Shorting and Leverage Constraints}

In the standard CPPI implementation there are no restrictions on either shorting the risky asset or on borrowing additional funds at the risk free rate. In practice however, restrictions on these actions are in place. In the extensions to the model described in this section, constraints are imposed on the CPPI to prevent shorting of the risky asset. This is achieved by ensuring that the exposure never becomes negative:

$$
C_{t_{k}}=\max \left[\left(V_{t_{k}}-F_{t_{k}}\right), 0\right]
$$

Additionally, constraints are placed on the amount of leverage (borrowing) that may be used. Defining $l$ as a multiple limiting the maximum exposure allowed, the value of the exposure is redefined as

$$
E_{t_{k}}=\min \left[m C_{t_{k}}, l V_{t_{k}}\right]
$$

Therefore $l=1$ implies that the portfolio is self-financing whilst $l=2$ allows a maximum leverage of $200 \%$ i.e. up to $100 \%$ of the portfolio's current value may be borrowed at the risk-free rate $r$. The maximum leverage permitted may be set by regulatory law, but regardless a maximum leverage of $200 \%$ is typically used in the industry e.g. Pain (2008).

\subsubsection{Ratchets}

In the original CPPI model, the floor is discounted back from maturity at the risk-free rate and is independent of the portfolio's performance. Due to the length of a typical CPPI investment being several years, previous gains in the portfolio value during strong periods of growth will be eroded in subsequent periods of decline. To help retain previous gains a ratchet or profit lock-in is commonly applied to the CPPI strategy. This serves to increase the terminal guarantee by increasing the value of the floor in reaction to increases in the portfolio value.

A ratcheting mechanism can be applied to the CPPI, allowing $100 \%$ of the initial investment value to be guaranteed with a floor that grows continuously at the risk-free rate. Equation (5) describes such a ratchet whereby the guarantee is increased by $\xi^{G} \%$ every time the portfolio value increases by a further $\xi^{V} \%$ step.

$$
\begin{aligned}
\Lambda_{t_{k}} & =\max \left(\text { floor }\left\{\frac{\left(V_{t_{k}} / V_{0}\right)-1}{\xi^{V}}\right\}, \Lambda_{t_{k-1}}\right) \\
\text { floor }(y) & =\max \{z \in \mathbb{Z} \mid z<y\} \\
g_{t_{k}} & =g_{0}+\Lambda_{t_{k}} \xi^{G} \\
G_{t_{k}} & =g_{t_{k}} V_{0} \\
F_{t_{k}} & =G_{t_{k}} e^{-r\left(T-t_{k}\right)} .
\end{aligned}
$$


The number of "clicks" of the ratchet applied up to time $t_{k}$ is denoted by $\Lambda_{t_{k}}$ with $\Lambda_{0}=0$. Since the multiplier value remains the same, the probability of the floor being hit remains the same.

The floor ratchet mechanism is illustrated with the following example. Consider the initial guarantee is equal to the initial investment which is $100\left(g_{0}=100 \%, G_{0}=V_{0}=100\right)$. The ratchet portfolio trigger $\xi^{V} \%$ is set to $10 \%$ and the guarantee increase value $\xi^{G} \%$ is set at $5 \%$. If during the investment period the portfolio value increases to 122 , then the ratchet will be clicked twice $($ floor $((122 / 100-1) / 0.1)=2)$ and the new guarantee value be 110 .

\section{Methodology}

We assess the performance of different CPPI strategies under a CPT utility function by evaluating their performance using Monte Carlo simulation of the price process employing a GJR-GARCH model (Glosten et al., 1993). The GJR model extends the standard GARCH model with an additional term that captures and assigns extra weight to negative returns. The $\operatorname{GJR}-\operatorname{GARCH}(P, Q)$ model is defined as

$$
\begin{aligned}
S_{t_{k}} & =S_{t_{k-1}} e^{R_{t_{k}}} \\
R_{t_{k}} & =K+\varepsilon_{t_{k}} \\
\varepsilon_{t_{k}} & =\sigma_{t_{k}} \eta_{t_{k}} \\
\sigma_{t_{k}}^{2} & =\omega+\sum_{q=1}^{Q} \alpha_{q} \varepsilon_{t_{k-q}}^{2}+\sum_{q=1}^{Q} \psi_{q} \varepsilon_{t_{k-q}}^{2} \mathbf{1}_{\left\{\varepsilon_{t_{k-q}}<0\right\}}+\sum_{p=1}^{P} \beta_{p} \sigma_{t_{k-p}}^{2}
\end{aligned}
$$

under the conditions

$$
\omega \geq 0, \quad \alpha_{q}, \beta_{p} \geq 0, \quad \alpha_{q}+\psi_{q} \geq 0, \quad \sum_{p=1}^{P} \beta_{p}+\sum_{q=1}^{Q} \alpha_{q}+\frac{1}{2} \sum_{q=1}^{Q} \psi_{q}<1,
$$

where $R_{t_{k}}$ is the daily $\log$-return, $\varepsilon_{t_{k}}$ is the unexpected return and $\sigma_{t_{k}}^{2}$ is the conditional variance at time $t_{k}$. $\eta_{t_{k}}$ is a random variable drawn from a Student's t distribution with $v$ degrees of freedom, zero mean and unit variance. The constant $K$ alters the expected value of the process. The model has been fitted to FTSE 100 data as described in A.

\section{Cumulative Prospect Theory}

Attempts to capture the preferences of investors, under uncertainty, had previously been formulated under the expected utility theory (EUT) framework. Under EUT investors are generally considered to be risk averse and have a concave value function. The expected utility value being determined through the linear probability weighting of utility values based on absolute values of wealth. However, EUT fails to capture the more complex behaviours that are apparent. For example, individuals exhibit loss aversion, nonlinear preferences and can be risk-seeking as well as risk-averse depending on the probability and value of outcomes (Kahneman and Tversky, 1979).

Prospect theory was introduced by Kahneman and Tversky (1979) to remedy apparent failures in EUT and was further developed as cumulative prospect theory (CPT) (Tversky and Kahneman, 1992). Prospect theory differs from expected utility theory in that it uses a reference point to distinguish between losses and gains. The value function is convex in the losses, exhibiting loss aversion and concave in the gains as in EUT. Additionally, actual probabilities are transformed, with the overweighting of small probabilities, to capture insurance and lottery type effects.

The same functional forms of CPT as used by Dichtl and Drobetz (2011) are also adopted here. However, it is defined as in the manner of Schmidt et al. (2008) where the reference point may be a 
stochastic value dependent on a realised state. Originally the reference point was assumed to be fixed and represent the status quo value. The value of an investment strategy $f$ dependent on a state $s_{i}$ is $f\left[s_{i}\right]$. The reference point $h$ generated under this state is $h\left[s_{i}\right]$. The outcome of a state is $x\left[s_{i}\right]=f\left[s_{i}\right]-h\left[s_{i}\right]$. The number of states is defined as $I=I^{+}+I^{-}$, with $I^{+}$being the number of positive or neutral outcomes and $I^{-}$the number of negative outcomes. Outcomes are ordered in ascending order such that $i>j$ if and only if $x\left[s_{i}\right]>x\left[s_{j}\right]$. The cumulative weighted prospect value is given as

$$
C P V(h)=\sum_{\substack{i=-I^{-} \\ i \neq 0}}^{I^{+}} v\left(x\left[s_{i}\right]\right) W\left(s_{i} ; f, h\right),
$$

where $v\left(x\left[s_{i}\right]\right)$ is the relative value under each state and $W\left(s_{i} ; f, h\right)$ is the weight applied to each state. As described earlier, the relative value function is a monotonically increasing function that is convex in losses and concave in gains, such that

$$
v(x)= \begin{cases}x^{\alpha} & \text { if } x \geq 0 \\ -\lambda(-x)^{\beta} & \text { if } x<0 .\end{cases}
$$

The ranked weights are formed from the following cumulative weighting scheme

$$
W\left(s_{i} ; f, h\right)= \begin{cases}w^{+}\left(\pi_{i}\right) & \text { if } i=I^{+}, \\ w^{+}\left(\sum_{j=i}^{I^{+}} \pi_{j}\right)-w^{+}\left(\sum_{j=i+1}^{I^{+}} \pi_{j}\right) & \text { if } 1 \leq i \leq I^{+}-1, \\ w^{-}\left(\sum_{j=-I^{-}}^{i} \pi_{j}\right)-w^{-}\left(\sum_{j=-I^{-}}^{i-1} \pi_{j}\right) & \text { if }-I^{-}+1 \leq i \leq-1, \\ w^{-}\left(\pi_{i}\right) & \text { if } i=-I^{-},\end{cases}
$$

using the weight function (Lattimore et al., 1992)

$$
w(p)=\frac{\delta p^{\gamma}}{\delta p^{\gamma}+(1-p)^{\gamma}}
$$

where $\delta, \gamma=\delta^{+}, \gamma^{+} \mid x \geq 0$ and $\delta, \gamma=\delta^{-}, \gamma^{-} \mid x<0$. The parameter values used are: $\alpha=\beta=0.88$; $\lambda=2.25 ; \delta^{+}=0.65 ; \delta^{-}=0.84 ; \gamma^{+}=0.6 ; \gamma^{-}=0.65$, which are the same values as used by Dichtl and Drobetz (2011).

In this application to portfolio insurance the investment strategy $f$ is $V_{T}^{G}$ with the outcome in (8) defined as:

$$
x\left[s_{i}\right]=V_{T}^{G}\left[s_{i}\right]-h\left[s_{i}\right]
$$

where $V_{T}^{G}$ is the terminal portfolio value from the perspective of the buyer, with the guarantee in place i.e. $V_{T}^{G}=\max \left(V_{T}, G_{T}\right)$. The determination of the reference point $h$ is discussed in the following subsection.

\subsection{Investor Reference Point}

As described in the previous subsection, a reference point is required to calculate the cumulative prospect value (CPV). The reference point is often assumed to be static and equal to the initial capital $V_{0}$ e.g. see Dichtl and Drobetz (2011). However, Arkes et al. (2008) suggests that this is not the case and in fact the investor is likely to adjust their reference point depending on stochastic outcomes of some benchmark. Furthermore, it is possible that the investor evaluates their performance based on multiple reference points. Koop and Johnson (2010) suggest three reference points representing the minimum acceptable value, the standard CPT reference point and the goal.

For the portfolio insurance investor, potential reference points include: the initial investment value $V_{0}$; the guarantee amount $G_{T}$ (equal to $V_{0}$ when no ratcheting occurs); the pure risk-free investment $V_{T}^{r f}$ i.e. 
$V_{0} e^{r T}$ or a CPPI strategy with $m=0$; the gapless portfolio $V_{T}^{m=1}$; the pure risky asset $S_{T}$; the maximum portfolio value achieved during the investment period $\max \left[V_{0 . . T}\right]$; the maximum risky asset value achieved during the investment period $\max \left[S_{0 . . T}\right]$. The first two points represent minimum acceptable values to the investor. The following three are simple alternative strategies in the investor's opportunity set that were not taken, but which the investor wants to outperform. The final two points attempt to capture past highs that the investor wishes to retain. The ratcheted floor can be considered an attempt to retain some amount of the value $\max \left[V_{0 . . T}\right]$.

As discussed in the introductions using multiple criteria to assess an investment is a complex area of research. In particular even if a suitable functional form is found, there is still the problem of eliciting parameter values. We apply a straightforward method whereby multiple reference points $h_{j}$ are combined into a single overall point $h^{*}$. In the simplest case $J$ reference points can be combined using a linear weighted sum

$$
h^{*}=\sum_{j=1}^{J} w_{j} h_{j},
$$

where $\sum_{j=1}^{J} w_{j}=1,0 \leq w_{j}$. Using this model it is possible for the investor to express a concrete preference (e.g. $50 \% V_{0}, 50 \% \max \left[V_{0 . . T}\right]$ ) before investment, but for an actual stochastic reference point value to be realised only at maturity.

There are two alternative formulations for combining the characteristics of multiple reference points without modifying the original reference point values. Firstly, the weighted linear sum can be applied to the independent values of the cumulative prospect value e.g. $w C P V\left(h_{1}\right)+(1-w) C P V\left(h_{2}\right)$. Secondly, a multi-objective Pareto optimal approach could be taken whereby non-dominated solutions are located. However, the creation of a single new reference point is the most parsimonious model.

\section{Results}

Unless stated otherwise the results are given without leverage i.e. $l=100 \%$. The risk-free rate of return $r$ is taken to be $4 \%$, the investment period is 5 years $(T=5)$ and daily trading $(n=1260)$ is assumed.

\subsection{Standard CPPI}

Figure 1 plots the cumulative prospect value of the CPPI, risky asset and risk-free portfolio with a reference point of $V_{0}$. It is shown that the risky asset is preferred over the riskless asset and that the CPPI is preferred for multiplier values greater than or equal to 2 . The CPT value is increasing with the multiplier value until $m=6$ and then declines slightly. The leverage constraint is responsible for limiting the increase in the CPT with $m$.

The effect of increasing levels of leverage are shown in Figure 2. As the maximum amount of leverage allowed increases, the increase in cumulative prospect value is substantial. This illustrates that the leverage constraint is a key factor in limiting the cumulative prospect value for multiplier values greater than 2.

\subsection{Ratcheting}

Given the popularity of ratcheting type mechanisms, an explanation is sought under the assumption that the investor uses cumulative prospect theory to value investments. A comparison of the standard and ratcheted CPPI under CPT is plotted in Figure 3. The graph clearly shows that using $V_{0}$ as the reference point, ratcheting is not favoured for all multiplier values. The greater the ratcheting effect i.e. the greater $\xi^{G}$, the worse the performance. This results suggests, for the parameter values considered at least, that ratcheting of the floor is not favoured by a CPT investor. This is likely due to the ratchet reducing 


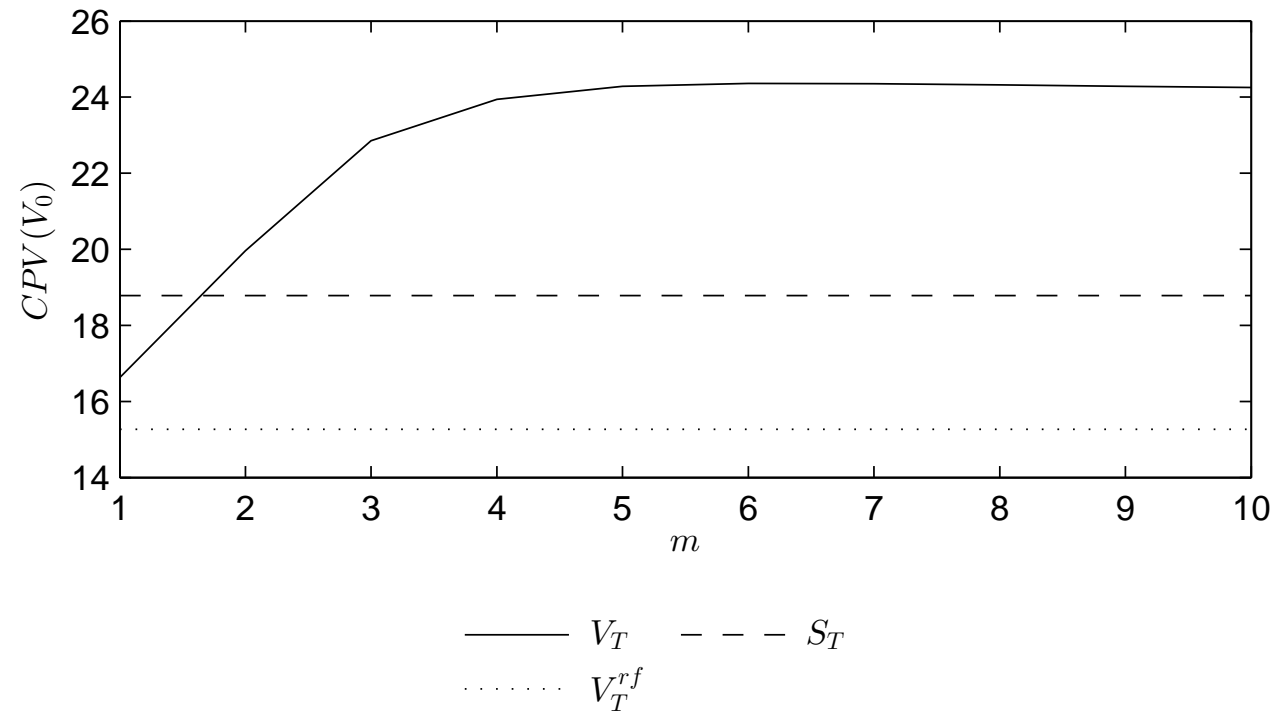

Figure 1: Cumulative prospect theory value with initial wealth $V_{0}$ as the reference point, for various multiplier values.

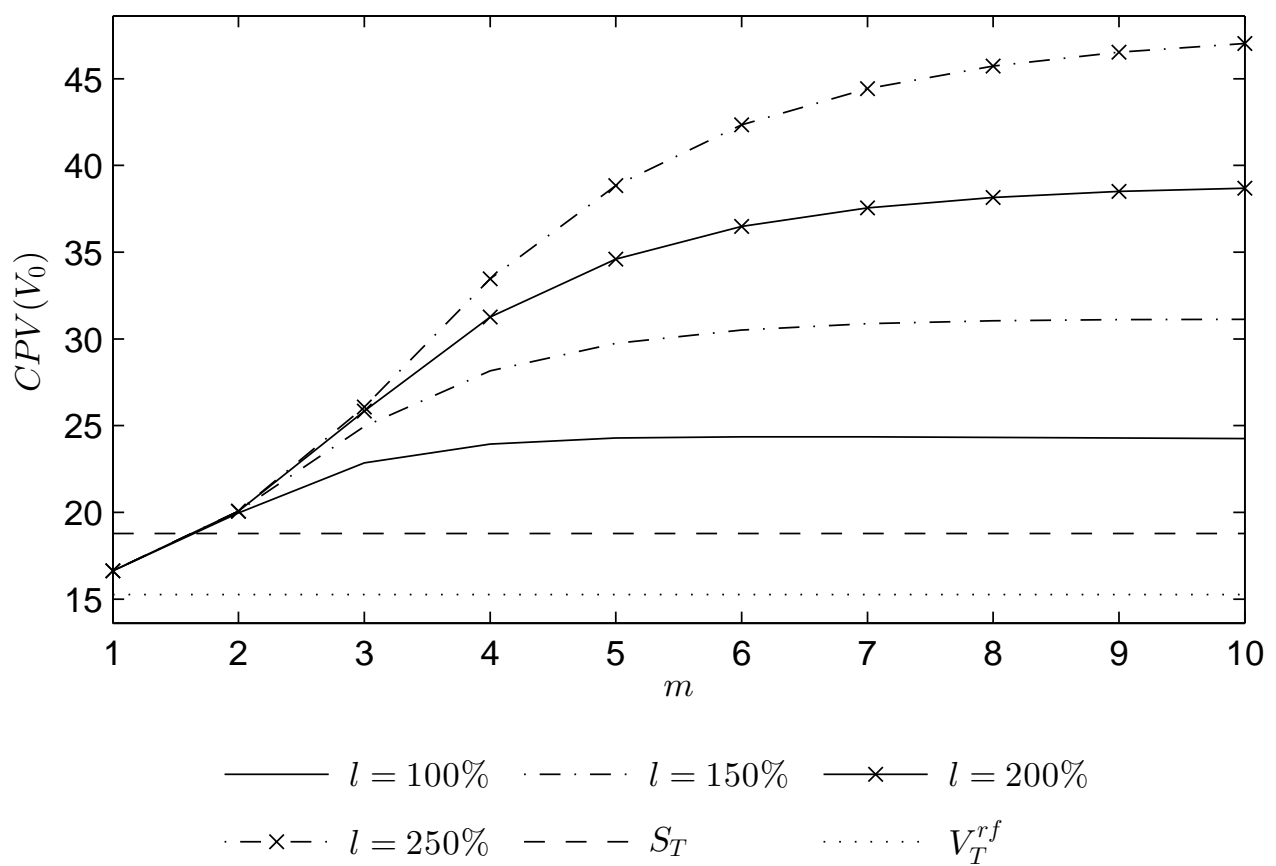

Figure 2: Cumulative prospect value with initial wealth $V_{0}$ as the reference point. Given for various multiplier values with maximum leverage values $(l)$ of $100 \%$ (no additional leverage), 150\%, 200\% and $250 \%$. 
exposure to the risky asset as it rises, limiting growth. Additionally, the greater guarantee value protects values larger than the initial investment, but since these values are already on the gain side of the CPT value function this provides little benefit.

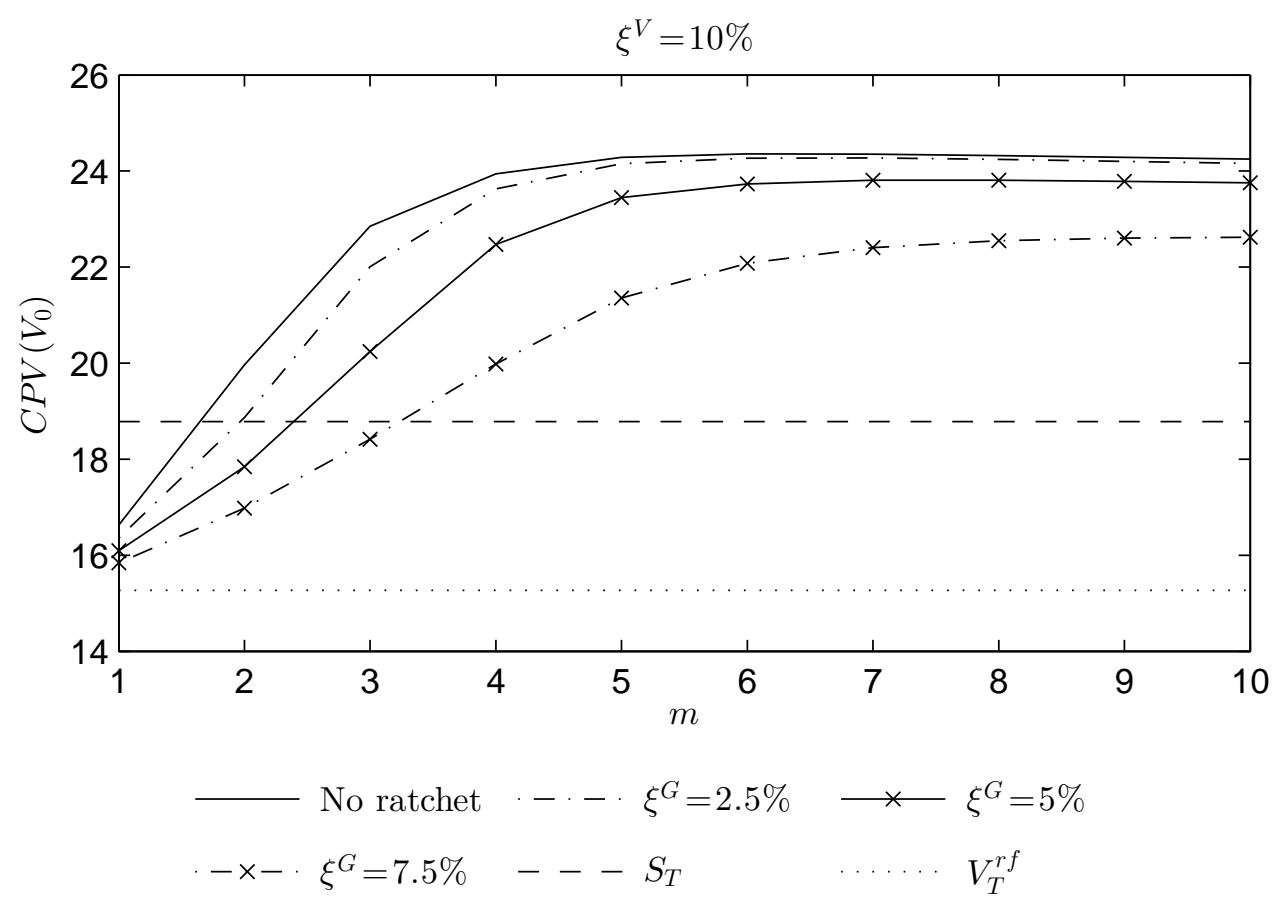

Figure 3: Cumulative prospect value with initial wealth $V_{0}$ as the reference point. Given for various multiplier values with a ratcheted floor with $\xi^{V}=10 \%$ and $\xi^{G}=2.5 \%, \xi^{G}=5 \%$ and $\xi^{G}=7.5 \%$.

Since one aspect of the ratchet's poor performance is due to it allocating capital to the risk-free asset when the risky asset rises, allowing extra leverage may improve its performance. Figure 4 plots the cumulative prospect value for two ratchet parameter sets with $\xi^{V}=10 \%$ and $\xi^{G}=2.5 \%, \xi^{G}=5 \%$ with maximum leverages of $150 \%$ and $200 \%$. Compared to the ratchet with no additional leverage in Figure 3 it is seen that leverage provides an increase in the cumulative prospect value. However, compared to the standard levered CPPI in Figure 2 the levered ratcheted strategy still performs significantly worse.

Previously the reference point has been assumed to be equal to the initial portfolio value. However, given the long duration of portfolio insurance investments which are typically around 5 years, it is unlikely that the investor's reference point remains at their initial wealth. Since the investor of portfolio insurance forgoes the certainty of the pure risk-free investment for the chance of greater returns from the risky asset, the risk-free portfolio value is an intuitive reference point. Additionally, since it grows with time it scales depending on the maturity of the investment. Figure 5 plots the ratcheted CPPI using the terminal risk-free portfolio value $V_{T}^{r f}$. For $m \geq 5$ the $\xi^{G}=2.5 \%$ and $\xi^{G}=5 \%$ ratchets slightly outperform the standard CPPI. This result indicates that a growing reference point may better describe the preference of an investor.

Given that the guarantee at the initial wealth protects a CPT investor from encountering the loss part of the value function, it would be intuitive to assume that the reference point adapts to equal the guarantee. Figure 6 plots the cumulative prospect value under a reference point of $G_{T}$ for $\xi^{V}=10 \%$ and $\xi^{G}=2.5 \%$, $\xi^{G}=5 \%$ and $\xi^{G}=7.5 \%$. Under $\xi^{V}=10 \%$ and $\xi^{G}=2.5 \%$ the ratchet portfolio value is increasing in 


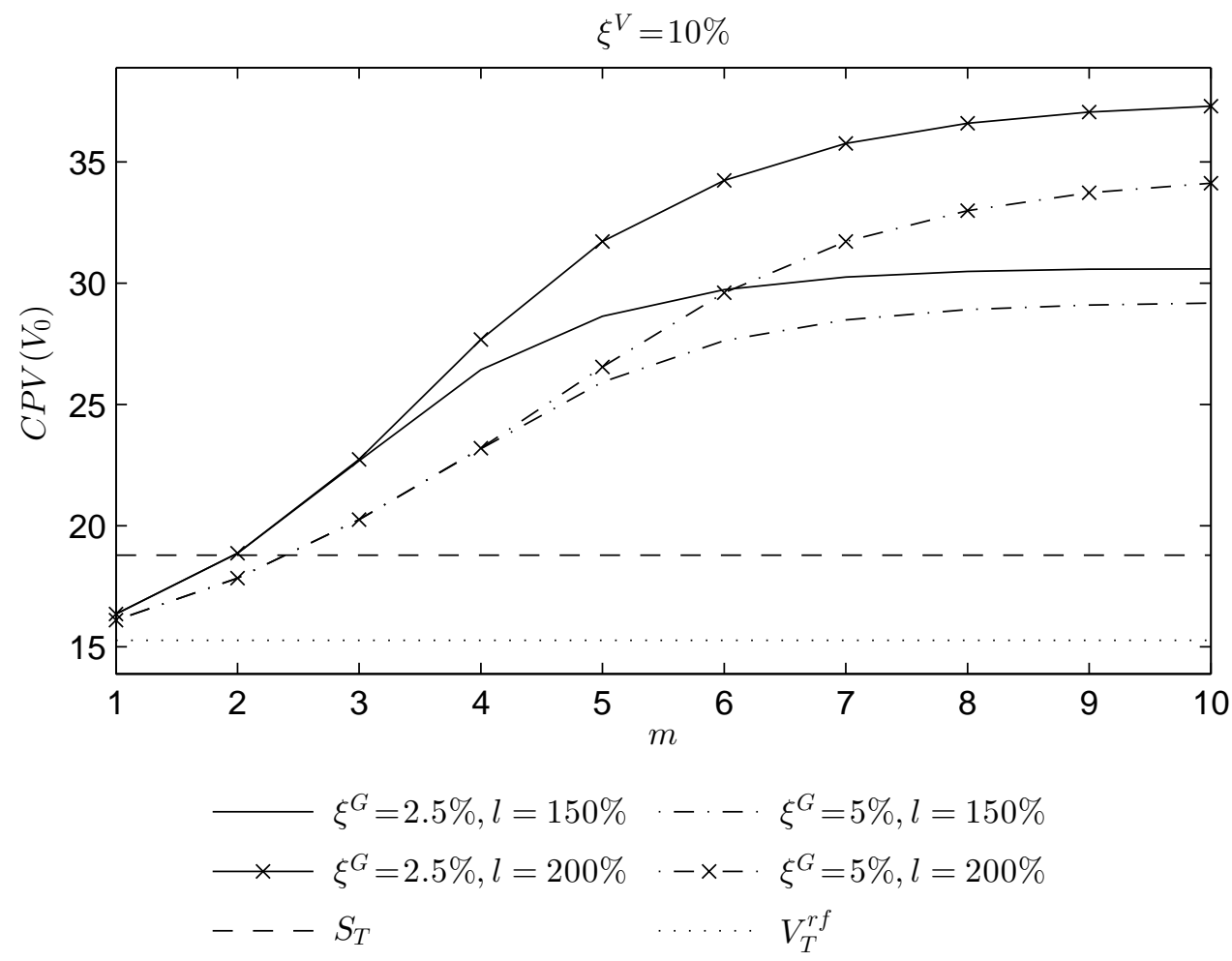

Figure 4: Cumulative prospect value with initial wealth $V_{0}$ as the reference point for various multiplier values with maximum leverage values $(l)$ of $150 \%$ and $200 \%$. Using a ratcheted floor with values $\xi^{V}=$ $10 \%$ and $\xi^{G}=2.5 \%, \xi^{G}=5 \%$ 


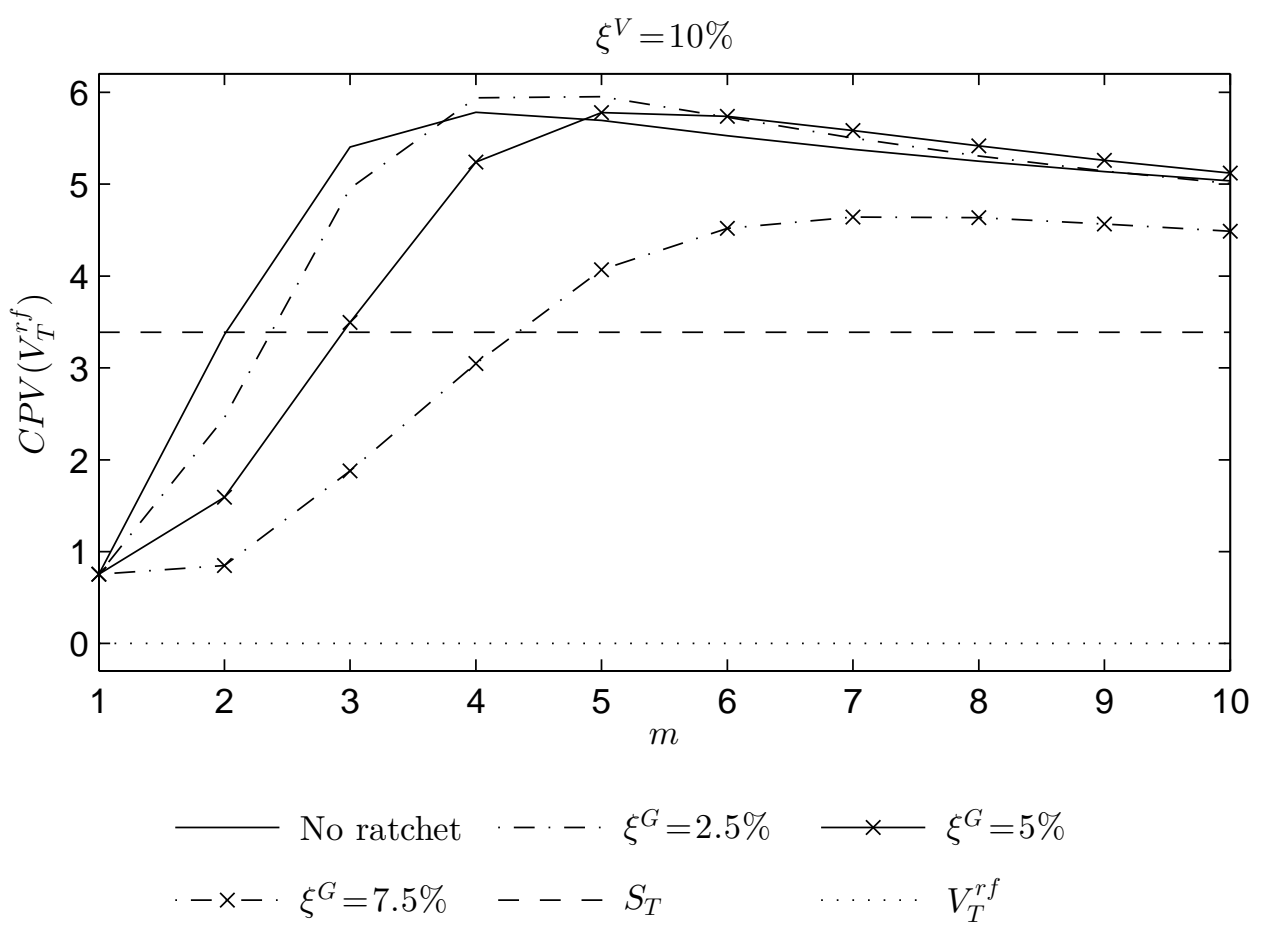

Figure 5: Cumulative prospect value with the risk-free portfolio value $V_{T}^{r f}$ as the reference point. Given for various multiplier values with a ratcheted floor with $\xi^{V}=10 \%$ and $\xi^{G}=2.5 \%, \xi^{G}=5 \%$ and $\xi^{G}=$ $7.5 \%$. 
$m$, while with $\xi^{V}=10 \%$ and $\xi^{G}=5 \%$ it is only increasing slightly and when $\xi^{V}=10 \%$ and $\xi^{G}=7.5 \%$ it decreases slightly. The limited increase $\left(\xi^{G}=2.5 \%, \xi^{G}=5 \%\right)$ or decrease $\left(\xi^{G}=7.5 \%\right)$ in CPV is partly due to the leverage constraint as described in the previous subsection. The other factor is the effect of the ratchet simultaneously increasing the guarantee, and hence the reference point, and reducing the exposure to the risky asset. This effect is most prominent when $\xi^{G}=7.5 \%$. The standard CPPI performs better than the ratcheted strategies for lower $m$, but then declines as $m$ increases. The better performance for lower multiplier values is due to the ratchet only coming into effect in very high growth scenarios for the risky asset. These scenarios benefit the standard CPPI more because their growth is not limited by the ratchet reducing exposure. As $m$ increases, many scenarios provide sufficient growth for the ratchets to click and subsequent drops in the risky asset value are protected against. For all of the ratchets, both the risk-free portfolio and risky asset are declining with $m$.

It has been implicitly assumed that the reference point of the investor follows the guarantee values as dictated by the ratcheted strategy. However, it should be noted that it is biased to compare the standard and ratcheted strategies using the ratcheted strategies' guarantee value as the reference point. Since it is assumed that the reference point follows the guarantee value as dictated by the strategy itself, then the standard CPPI can only be evaluated with a reference point of $G_{T}=V_{0}$ (since $g_{0 . . T}=100 \%$ ). In the following subsection it is argued that ratchets attempt to capture a more general behaviour of investors and under this generalisation they are preferable to investors.

\subsection{Investor Preferences and Reference Point}

As the previous results have shown, a CPT investor would not choose to use a ratcheted CPPI over the unratcheted strategy if their reference point is fixed at their initial wealth $\left(V_{0}\right)$. However, this behaviour is inconsistent with the popularity of ratchets leading to the proposition of an adaptive reference point. Furthermore, since the ratchet seeks to retain the past gains of the portfolio, this can be generalised to describe the behaviour of the investor as seeking to retain the best ever portfolio value $\max \left[V_{0 . . T}\right]$. The reference point $h^{*}$ is then defined as

$$
h^{*}=(1-w) V_{0}+w \max \left[V_{0 . . T}\right]
$$

where $0 \leq w \leq 1$ is the percentage weight preference an investor has towards the maximum portfolio value observed of a particular strategy i.e. $h_{1}=V_{0}$ and $h_{2}=\max \left[V_{0 . . T}\right]$ in Equation (13). When $h_{2}=$ $\max \left[V_{0 . . T}\right]$ it is assumed that the investor strictly compares the performance of the strategy to its current maximum observed value, and is ignorant of the performance of other strategies including the risk-free and risky asset. In this subsection a weight preference of $w=50 \%$ has been chosen to demonstrate an investor who has a reference point located at the midpoint between their initial wealth and the maximum observed portfolio value. Three sets of ratchet parameters: $\xi^{V}=5 \%, \xi^{G}=2.5 \% ; \xi^{V}=10 \%, \xi^{G}=5 \%$; $\xi^{V}=15 \%, \xi^{G}=7.5 \%$, have been selected to reflect that $50 \%$ of the wealth should be protected. This is in line with the premise that it is beneficial for the guarantee to equal the reference point, as proposed in the previous subsection.

Figure 7 compares the CPV of the unratcheted CPPI, ratcheted CPPI, risk-free portfolio and risky asset. The risky asset performs the worst, reflecting its higher volatility which allows it to attain high maximum values, but not retain them. The unratcheted CPPI also performs poorly due to it not possessing any mechanism to retain past maximums. This effect is more pronounced for higher multiplier values where higher maximum values are achieved. Since the risk-free portfolio grows at a constant rate to maturity it always achieves its maximum value giving it a positive $\mathrm{CPV}$ for $w<100 \%$. The $\xi^{V}=$ $5 \%, \xi^{G}=2.5 \%$ ratchet is the best performing strategy followed by the $\xi^{V}=10 \%, \xi^{G}=5 \%$ and $\xi^{V}=$ $15 \%, \xi^{G}=7.5 \%$ ratchets. The more sensitive the ratchet the better it performs because it more rapidly protects each new maximum level of wealth. The CPV values of the ratcheted strategies peak between 

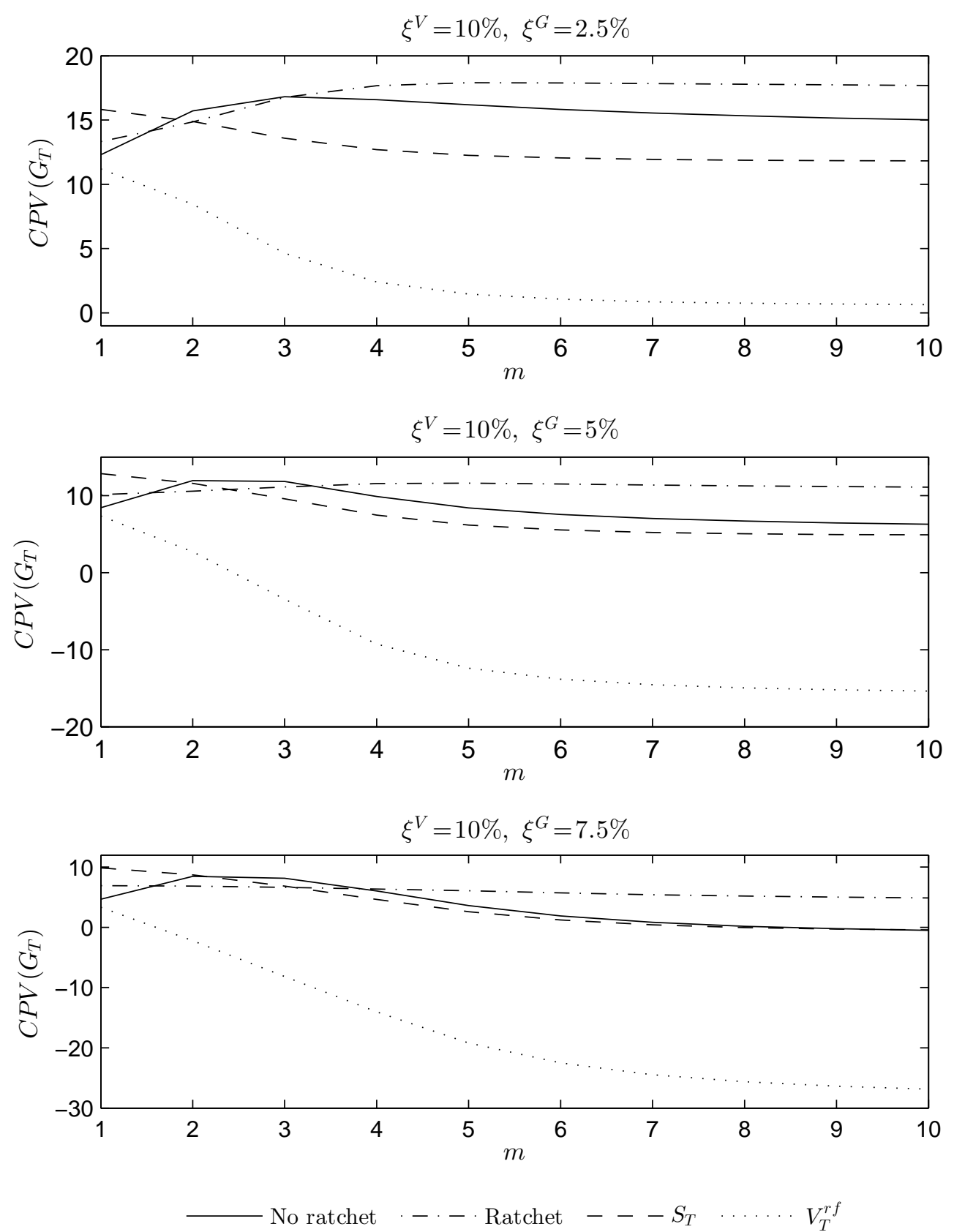

Figure 6: Cumulative prospect value with the guarantee level $G_{T}$, as determined by the ratcheted model, as the reference point. Given for various multiplier values with a ratcheted floor with $\xi^{V}=10 \%$ and $\xi^{G}=2.5 \%, \xi^{G}=5 \%$ and $\xi^{G}=7.5 \%$. 
$m=4$ and $m=5$ and then decline for higher multiplier values. This decline is caused by growth in the portfolio increasing the maximum value before the ratchet can be clicked.

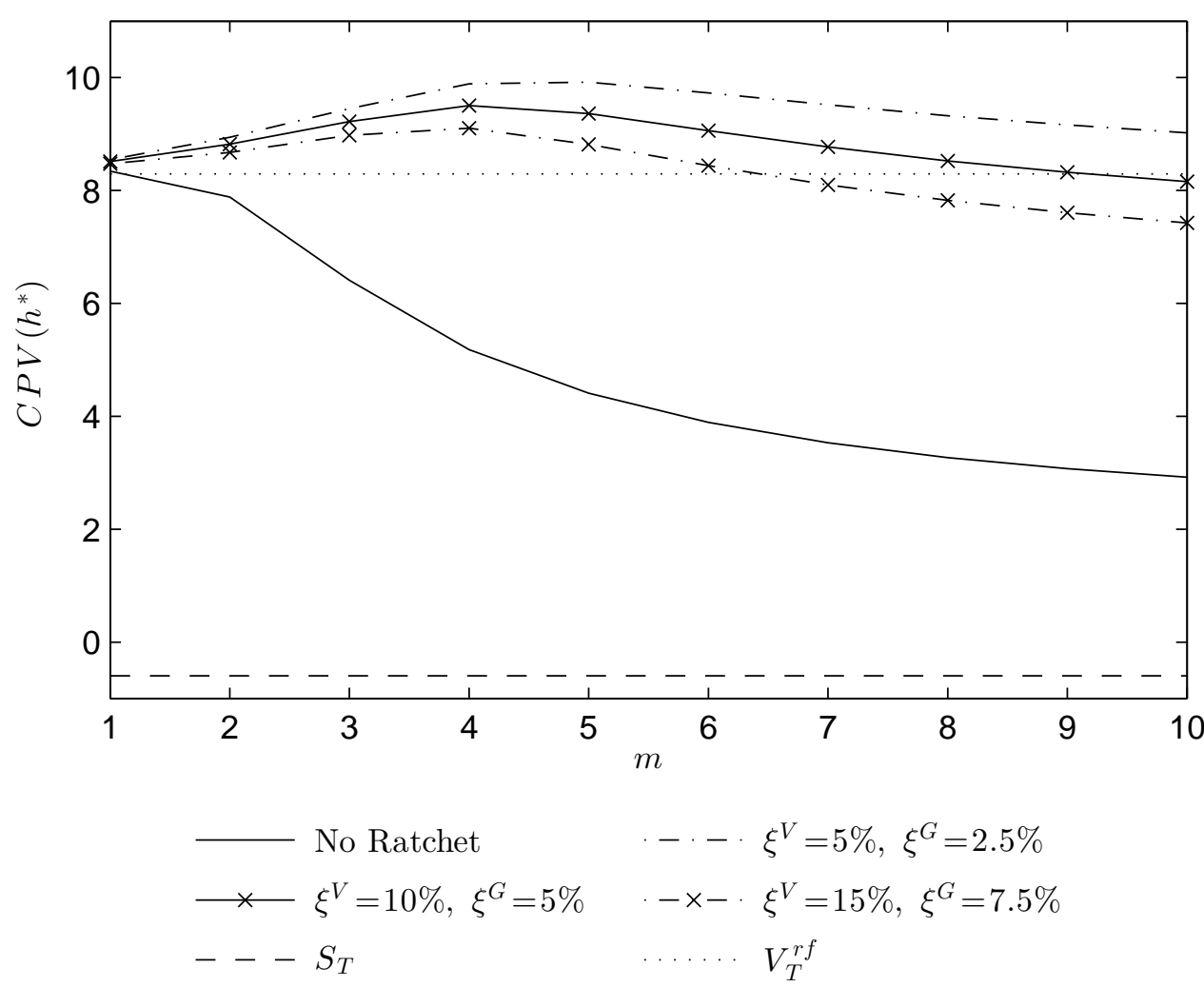

Figure 7: Cumulative prospect value using a reference point of $w=50 \%, h_{1}=V_{0}$ and $h_{2}=\max \left[V_{0 . . T}\right]$. Given for various multiplier values and ratcheted and unratcheted CPPI.

The impact of $200 \%$ maximum leverage is shown in Figure 8. Compared to Figure 7 where no additional leverage is allowed, the unratcheted CPPI is seen to perform even worse owing to even greater maximum values obtained. The ratcheted strategies all perform better, benefiting from the additional growth provided by the leverage with higher multiplier values.

The previous two figures assumed that the investor used the maximum observed value of a strategy to evaluate that particular strategy. However, as evident from Equation (14), this leads to an issue in strategies with lower terminal portfolio values, but also lower maximums, potentially resulting in higher payoffs i.e. the ratchet allows the terminal portfolio to get closer to the maximum because it limits growth resulting in a lower maximum than what the unratcheted CPPI would achieve. Given that we seek to investigate whether augmenting the standard CPPI with a ratchet is actually beneficial, the reference point $h^{*}$ can be redefined as

$$
h^{*}=(1-w) V_{0}+w \max \left[V_{0 . . T}^{\text {stand }}\right],
$$

where $\max \left[V_{0 . . T}^{\text {stand }}\right]$ is the maximum observed value of the standard unratcheted strategy i.e. the CPPI strategy with the constant rate floor. We can then fairly assess if it is beneficial for the investor to switch to the ratcheted variant of the CPPI. 


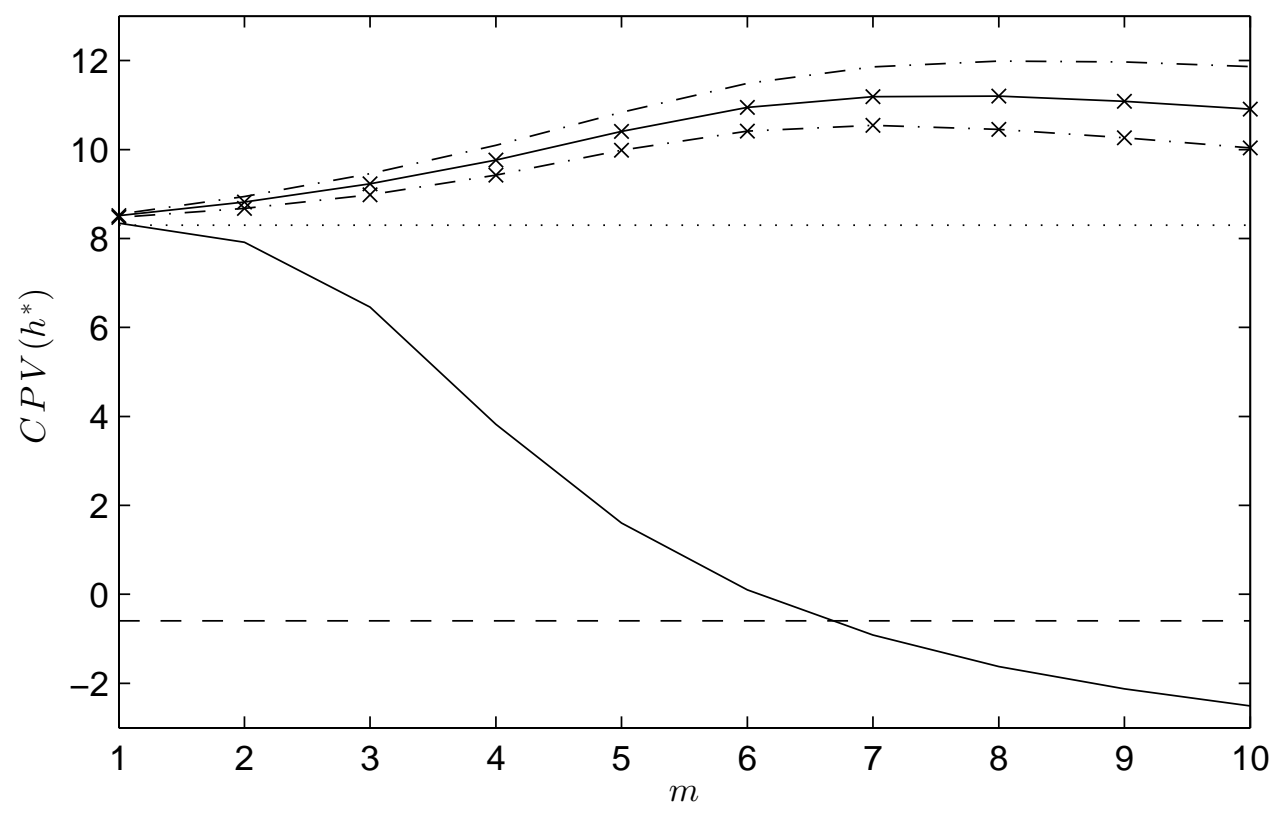

$$
\begin{aligned}
& \text { - No Ratchet } \quad-\cdot-\xi^{V}=5 \%, \xi^{G}=2.5 \% \\
& \longrightarrow \xi^{V}=10 \%, \xi^{G}=5 \% \quad \cdot-\times-\cdot \xi^{V}=15 \%, \xi^{G}=7.5 \% \\
& --S_{T} \quad \cdots \cdots V_{T}^{r f}
\end{aligned}
$$

Figure 8: Cumulative prospect value using a reference point of $w=50 \%, h_{1}=V_{0}$ and $h_{2}=\max \left[V_{0 . . T}\right]$. Given for various multiplier values and ratcheted and unratcheted CPPI with $200 \%$ maximum leverage $(l=200 \%)$. 
Figure 9 compares the different strategies for multiplier values of 1 to 10 . In contrast to all previous figures the results cannot be compared across multiplier values, but only between strategies for a particular multiplier value. In essence it is assumed that the multiplier value has already been chosen and the decision to be made is which strategy to select. The figure shows that the risk-free portfolio performs very poorly since it cannot adjust to the maximum value achieved by the CPPI. The risky asset performs well for lower multiplier values, but less so for $m>4$. When $m$ is low the maximum values are likely to be low so there would be many instances of $S_{T}>h^{*}$. However, for higher multiplier values this would not be the case. The ratcheted strategies perform best for $m \geq 4$. For $m<4$ the unratcheted CPPI performs better because the ratchets greatly restrict growth, whereas when $m \geq 4$ there is sufficient exposure to grow and retain past gains.

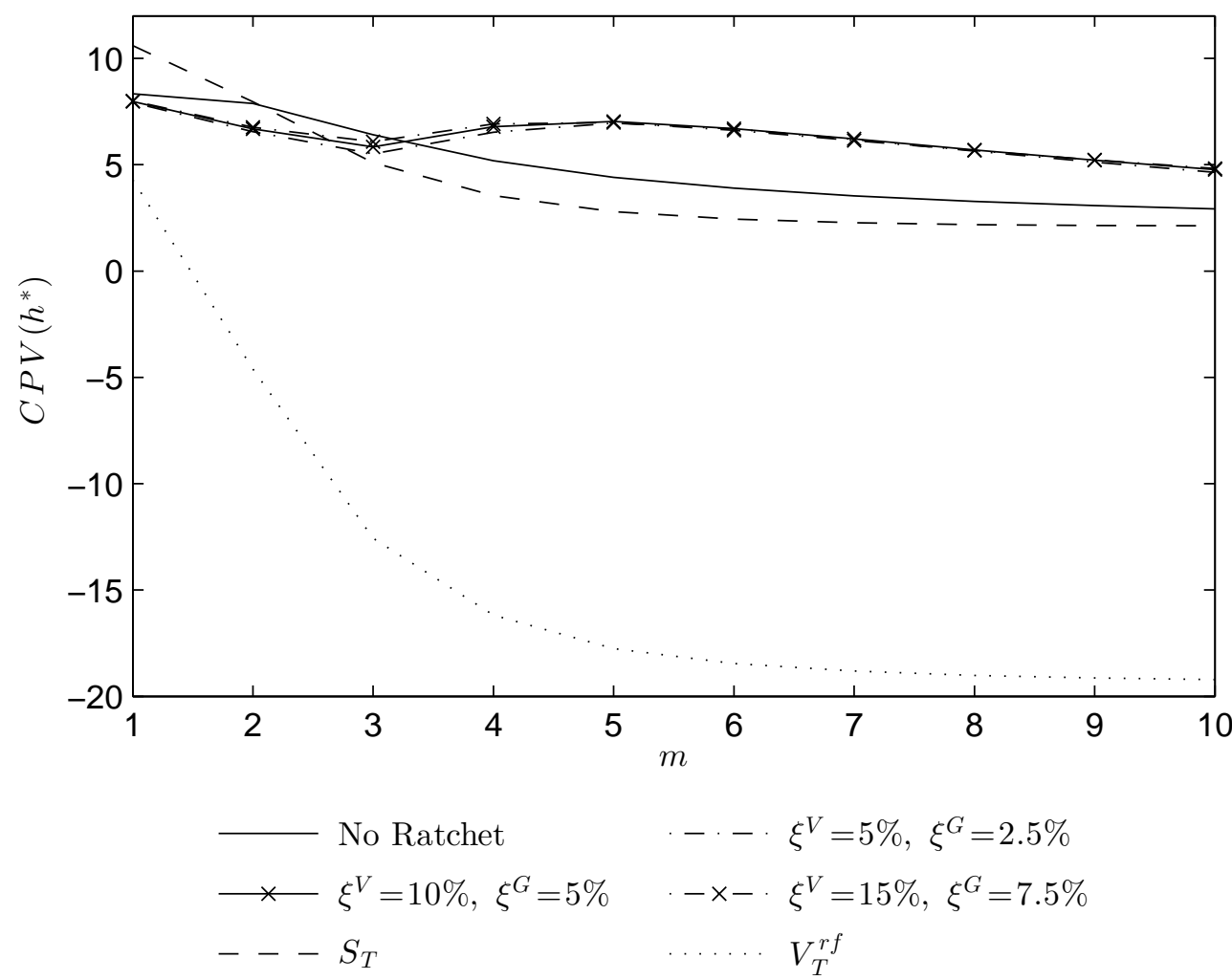

Figure 9: Cumulative prospect value using a reference point of $w=50 \%, h_{1}=V_{0}$ and $h_{2}=\max \left[V_{0 . . T}^{\text {stand }}\right]$. Given for various multiplier values and ratcheted and unratcheted CPPI.

In Figure (10) a maximum leverage of $200 \%$ is permitted. The impact of this is that the ratcheted strategies are unable to outperform the standard CPPI for any of the multiplier values tested. This is due to the ratchets limiting growth too greatly to match the higher maximum values attained by the unratcheted CPPI. Further evidence of this is seen in the ordering of the ratcheted strategies. The $\xi^{V}=15 \%, \xi^{G}=$ $7.5 \%$ ratchet performs best followed by the $\xi^{V}=10 \%, \xi^{G}=5 \%$ and the $\xi^{V}=5 \%, \xi^{G}=2.5 \%$. Since the $\xi^{V}=15 \%, \xi^{G}=7.5 \%$ ratchet clicks less often it inhibits growth less and therefore performs better. However, given that the reference point is dependent on the standard CPPI strategy, the ratchet parameters tested may perform best when $w \neq 50 \%$. This effect is more apparent when leverage is permitted, as 
demonstrated later. Figure 11 plots the strategies for $w=40 \%$. When $m \geq 7$ the ratcheted strategies are able to outperform the standard CPPI.

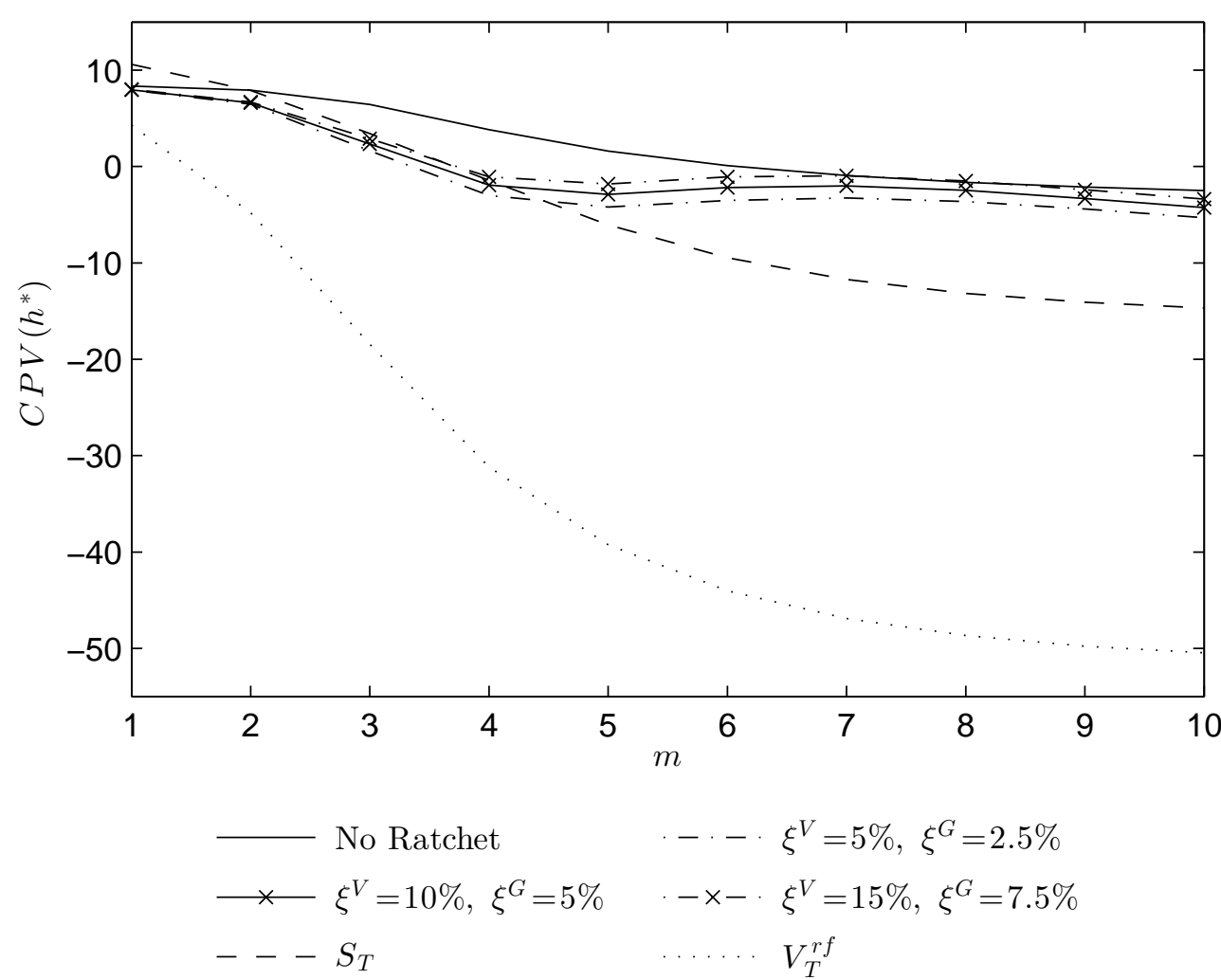

Figure 10: Cumulative prospect value using a reference point of $w=50 \%, h_{1}=V_{0}$ and $h_{2}=\max \left[V_{0 . . T}^{\text {stand }}\right]$. Given for various multiplier values and ratcheted and unratcheted CPPI with 200\% maximum leverage $(l=200 \%)$.

Figures 10 and 11 demonstrated that a ratchet designed to protect $50 \%$ of the maximum wealth may not specifically suit an investor with a $w=50 \%$. In the following two figures: Figure 12 and Figure 13, the difference in CPV between the standard CPPI and a ratcheted $\xi^{V}=10 \%, \xi^{G}=5 \%$ strategy is shown for $w$ values from $0-100 \%$. Figure 12 clearly shows that the ratcheted CPPI is preferred for a broad area around $m=6$ and a weighting of 50\%. The effect of $200 \%$ maximum leverage is shown in Figure 13. Compared to Figure 12, the area where the ratcheted CPPI is preferred is shifted down and to the right. The difference is also significantly smaller. A $\xi^{V}=10 \%, \xi^{G}=5 \%$ ratchet would benefit an investor with $w=40 \%$ more than one with $w=50 \%$ when leverage is permitted because the unratcheted strategy attains higher maximums. The ratcheted strategy reduces exposure and growth resulting in it protecting only $40 \%$ rather than $50 \%$ of the unratcheted CPPI's maximum.

When there is no leverage a trough is present for multiplier values between 2 and 3 . With a maximum leverage of $200 \%$ there is a trough between 3 and 4 . In both cases there is a larger negative value when there is a higher preference weighting. This feature can be explained as follows. The CPV increases most under a CPPI strategy when there is strong growth and therefore a high payoff. However, for higher multiplier values the leverage constraint prevents increased investment in the risky asset resulting in $100 \%$ 


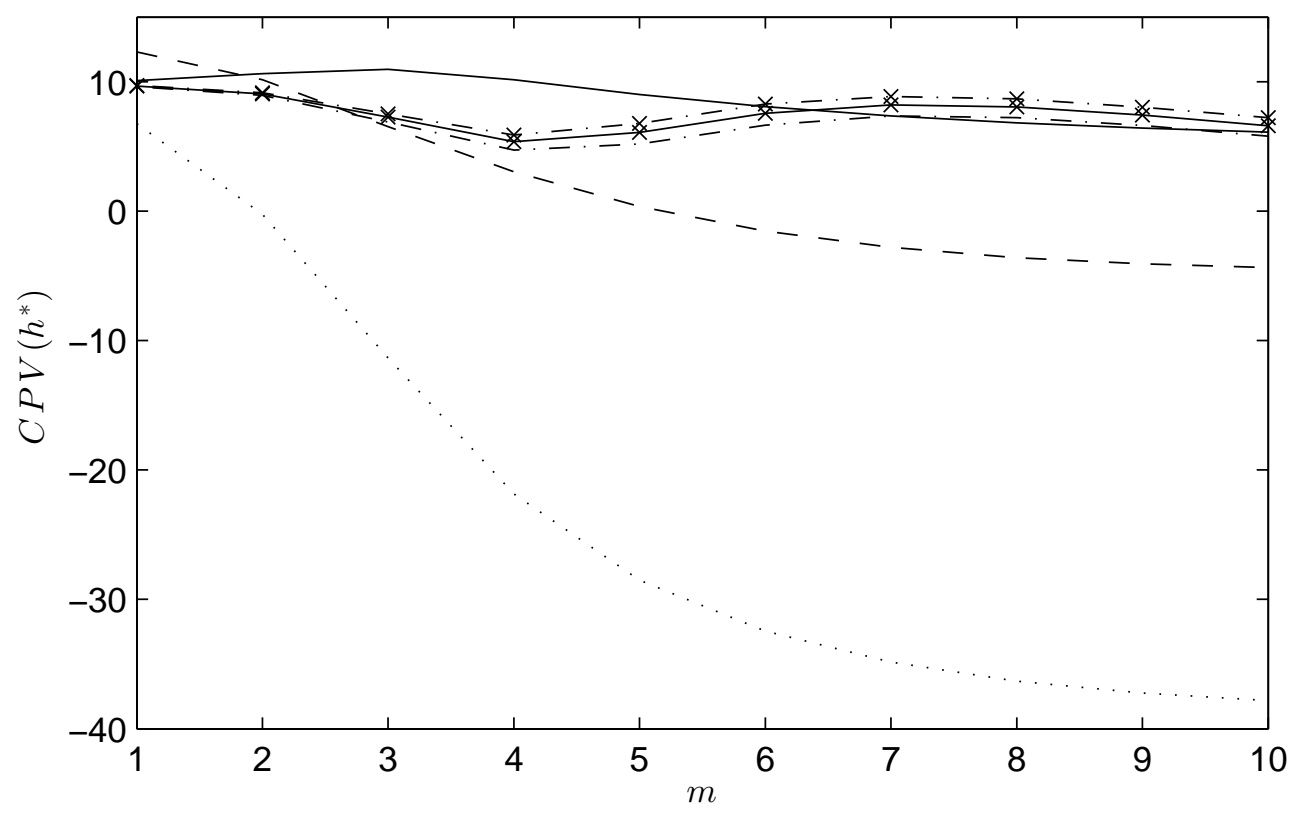

$$
\begin{aligned}
& \text { - No Ratchet } \quad-\cdot-\xi^{V}=5 \%, \xi^{G}=2.5 \% \\
& \longrightarrow \xi^{V}=10 \%, \xi^{G}=5 \% \quad \cdot-\times-\cdot \xi^{V}=15 \%, \xi^{G}=7.5 \% \\
& --S_{T} \quad \cdots \cdots V_{T}^{r f}
\end{aligned}
$$

Figure 11: Cumulative prospect value using a reference point of $w=40 \%, h_{1}=V_{0}$ and $h_{2}=\max \left[V_{0 . T}^{\mathrm{stand}}\right]$. Given for various multiplier values and ratcheted and unratcheted CPPI with $200 \%$ maximum leverage $(l=200 \%)$. 


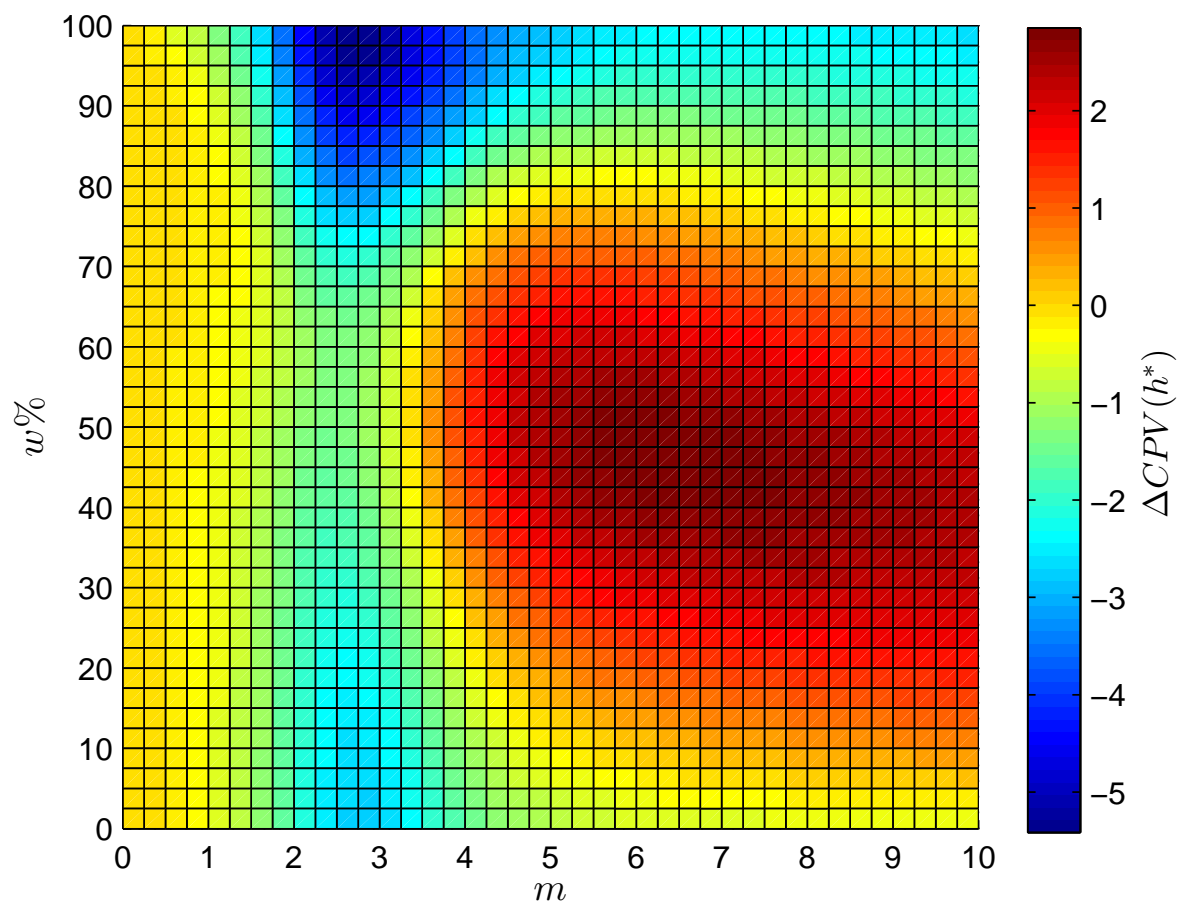

Figure 12: Difference in cumulative prospect value between ratcheted $\left(\xi^{V}=10 \%, \xi^{G}=5 \%\right)$ and unratcheted CPPI for an investor with $h_{1}=V_{0}$ and $h_{2}=\max \left[V_{0 . T}^{\text {stand }}\right]$. 


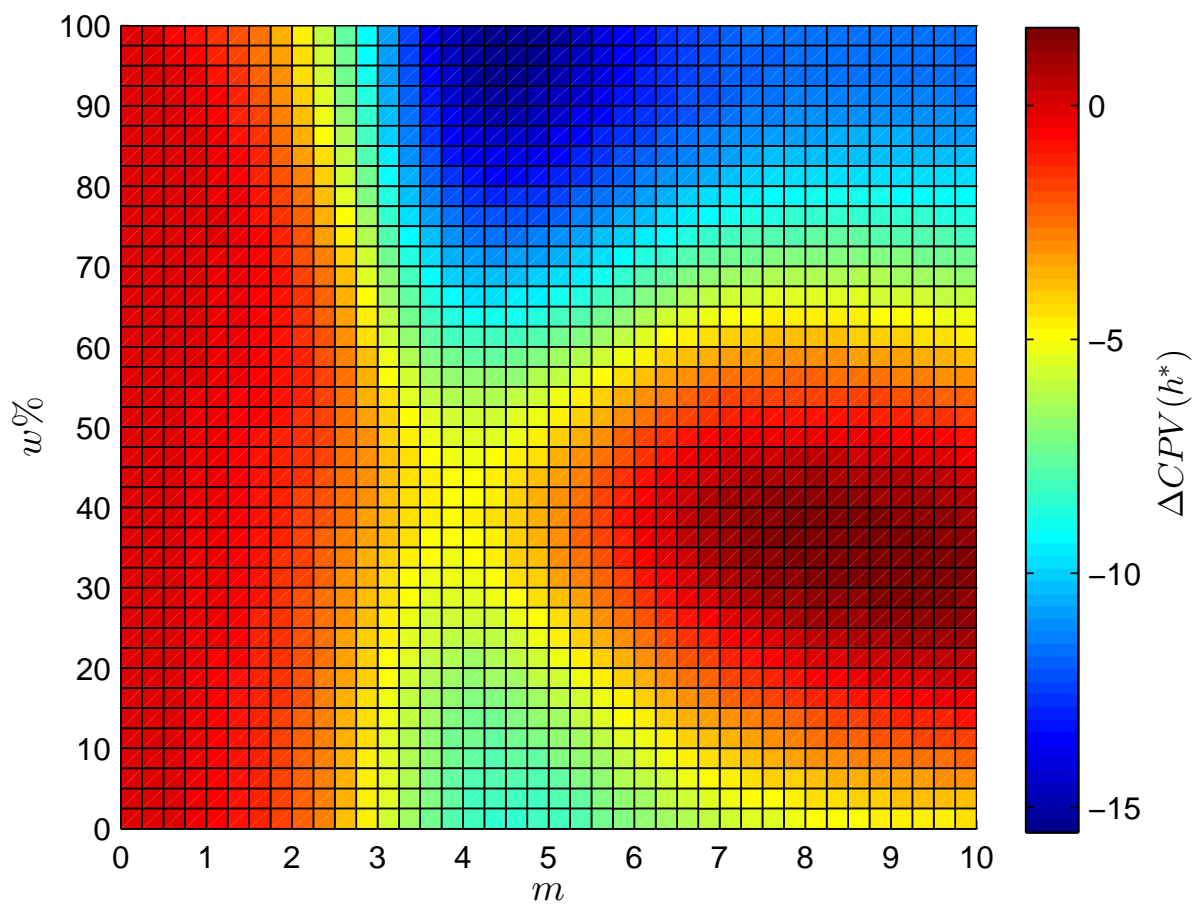

Figure 13: Difference in cumulative prospect value between ratcheted $\left(\xi^{V}=10 \%, \xi^{G}=5 \%\right)$ and unratcheted CPPI for an investor with $h_{1} V_{0}$ to $h_{2} \max \left[V_{0 . . T}^{\text {stand }}\right]$ with $200 \%$ leverage $(l=200 \%)$. 
(or 200\% for the levered approach) investment in S. As demonstrated in Subsection 5.1, increasing the leverage causes a large increase in the CPV so the leverage constraint is a big limiting factor. This affects both the standard and ratcheted strategies. However, for the lower multiplier values, where the troughs occur, the leverage constraint does not have much impact. Instead the ratcheting of the floor is the biggest inhibitor of growth because capital is allocated away from the risky asset to the risk-free asset. The result is that the standard CPPI outperforms the ratcheted CPPI.

\section{Conclusion}

Constant proportion portfolio insurance is a widely used investment strategy. Dichtl and Drobetz (2010, 2011) support the view that investors of CPPI have a preference structure adequately described by cumulative prospect theory. However, a conflict occurs with the introduction of a ratcheted floor. Ratcheted floor products (including time-invariant portfolio protection) are extensions (alternatives) to the CPPI that are popular with investors. Under CPT however, our results have show that a ratcheted floor is not preferred over the standard CPPI. This is because the ratchet protects increasing levels of wealth while the reference point remains static at the initial wealth value. Since the ratchet limits upside growth in order to protect greater wealth levels it is not rewarded under the standard fixed reference point cumulative prospect theory.

In this paper we have proposed an extension to CPT accommodate both the ideas that investors use CPT, but also like ratcheted investments. We have proposed that the investor does not have a fixed reference point, but rather it adapts over time. Specifically, the investor uses a linear combination of their initial wealth $V_{0}$ and the current observed maximum risky price value $\max \left[V_{0 . . T}\right]$. The evidence in this paper supports our proposed framework with the investor with an adaptive reference point preferring the ratcheted CPPI over the plain model in some cases. More generally our results suggest that the CPT investor prefers to have the guarantee minimum payoff match their reference point. This allows the loss part of the value function to be completely ignored producing a higher prospect theory value.

\section{References}

Arkes, H., Hirshleifer, D., Jiang, D., Lim, S., Jan. 2008. Reference point adaptation: Tests in the domain of security trading. Organizational Behavior and Human Decision Processes 105 (1), 67-81.

Balder, S., Brandl, M., Mahayni, A., 2009. Effectiveness of CPPI strategies under discrete-time trading. Journal of Economic Dynamics \& Control 33 (1), 204-220.

Basak, S., Jul. 2002. A comparative study of portfolio insurance. Journal of Economic Dynamics and Control 26 (7-8), 1217-1241.

Ben Ameur, H., 2010. GARCH Models with CPPI Application. In: Jawadi, F., Barnett, W. A. (Eds.), Nonlinear Modeling of Economic and Financial Time-Series. (International Symposia in Economic Theory and Econometrics, Volume 20. Emerald Group Publishing Limited, pp. 187-205.

Ben Ameur, H., Prigent, J.-1., 2011. CPPI Method with a Conditional Floor. International Journal of Business 16 (3), 218-229.

Bertrand, P., Prigent, J.-1., 2002. Portfolio Insurance the extreme value approach to the CPPI method. Finance 23 (2), 69-86.

Black, F., Jones, R. C., 1987. Simplifying portfolio insurance. Journal of Portfolio Management 14 (1), 48-51. 
Black, F., Perold, A. F., 1992. Theory of constant proportion portfolio insurance. Journal of Economic Dynamics \& Control 16 (3-4), 403-426.

Boulier, J.-F., Kanniganti, A., 1995. Expected Performance and Risks of Various Portfolio Insurance Strategies. In: 5th AFIR Colloquium. Brussels, Belgium, pp. 1093-1124.

Branger, N., Mahayni, A., Schneider, J. C., Jun. 2010. On the optimal design of insurance contracts with guarantees. Insurance: Mathematics and Economics 46 (3), 485-492.

Chen, J.-S., Chang, C.-L., Hou, J.-L., Lin, Y.-T., 2008. Dynamic proportion portfolio insurance using genetic programming with principal component analysis. Expert Systems with Applications 35, 273278.

Choie, K. S., Seff, E. J., 1989. TIPP: Insurance without complexity: Comment. The Journal of Portfolio Management 16 (1), 107-108.

Cont, R., Tankov, P., Jul. 2009. Constant Proportion Portfolio Insurance in the Presence of Jumps in Asset Prices. Mathematical Finance 19 (3), 379-401.

Dichtl, H., Drobetz, W., 2010. On the Popularity of the CPPI Strategy: A Behavioral-Finance-Based Explanation and Design Recommendations. The Journal of Wealth Management 13 (2), 41-54.

Dichtl, H., Drobetz, W., Dec. 2011. Portfolio insurance and prospect theory investors: Popularity and optimal design of capital protected financial products. Journal of Banking \& Finance 35 (7), 16831697.

Dierkes, M., Erner, C., Zeisberger, S., May 2010. Investment horizon and the attractiveness of investment strategies: A behavioral approach. Journal of Banking \& Finance 34 (5), 1032-1046.

Do, B. H., 2002. Relative Performance of Dynamic Portfolio Insurance Strategies: Australian Evidence. Accounting and Finance 42 (3), 279-296.

Do, B. H., Faff, R. W., Jun. 2004. Do Futures-Based Strategies Enhance Dynamic Portfolio Insurance? Journal of Futures Markets 24 (6), 591-608.

Estep, T., Kritzman, M., 1988. TIPP: Insurance without complexity. The Journal of Portfolio Management 14 (4), 38-42.

Glosten, L. R., Jagannathan, R., Runkle, D. E., 1993. On the Relation Between the Expected Value and the Volatility of the Nominal Excess Return on Stocks. Journal of Finance 48 (5), 1779-1801.

Hamidi, B., Jurczenko, E., Maillet, B., 2009. A CAViaR Time-Varying Proportion Portfolio Insurance. Bankers, Markets \& Investors September- (102), 4-21.

Jessen, C., 2010. Constant Proportion Portfolio Insurance : Discrete-time Trading and Gap Risk Coverage. In: 23rd Australasian Finance and Banking Conference 2010. Copenhagen Business School Department of Finance, pp. 1-24.

Kahneman, D., Tversky, A., Mar. 1979. Prospect Theory: An Analysis of Decision under Risk. Econometrica 47 (2), 263.

Koop, G. J., Johnson, J. G., 2010. The Use of Multiple Reference Points in Risky Decision Making. Journal of Behavioral Decision Making 25 (1), 49-62.

Lattimore, P. K., Baker, J. R., Witte, A. D., 1992. The influence of probability on risky choice: A parametric examination. Journal of Economic Behavior \& Organization 17 (3), 377-400. 
Lee, H.-I., Hsu, H., Chiang, M.-H., Nov. 2010. Portfolio insurance with a dynamic floor. Journal of Derivatives \& Hedge Funds 16 (3), 219-230.

Lin, C., Huang, W., Zeelenberg, M., Dec. 2006. Multiple reference points in investor regret. Journal of Economic Psychology 27 (6), 781-792.

Maringer, D., Ramtohul, T., 2011. GP-based rebalancing triggers for the CPPI. In: Computational Intelligence for Financial Engineering and Economics (CIFEr), 2011 IEEE Symposium on. pp. 1-8.

Pain, D., 2008. Recent developments in portfolio insurance. Bank of England Quarterly Bulletin Q1 (Research and Analysis), 37-46.

Perold, A. F., Sharpe, W. F., 1988. Dynamic Strategies for Asset Allocation. Financial Analysts Journal (February), 16-27.

Schmidt, U., Starmer, C., Sugden, R., May 2008. Third-generation prospect theory. Journal of Risk and Uncertainty 36 (3), 203-223.

Tversky, A., Kahneman, D., 1992. Advances in Prospect Theory: Cumulative Representation of uncertainty. Journal of Risk and Uncertainty 5 (4), 297-323.

Vrecko, D., Branger, N., 2009. Why is portfolio insurance attractive to investors? http://ssrn.com/abstract=1519344.

\section{A Time Series Statistics}

\section{A.1 Model Parameters}

A GJR-GARCH(1,1) model with Student-t distributed innovations was fitted to daily log-return data from the FTSE 100 index, from $8^{\text {nd }}$ March 1990 to $15^{\text {th }}$ December 2010 inclusively. Parameter estimation and simulations were undertaken using the MATLAB Garch Toolbox. Simulations were of daily log-returns for 5 years (1260 realisations) across $10^{6}$ paths. Table 1 gives the model parameter values and their errors. Test statistics for the innovations are given in Table 2, confirming the null hypothesis that the innovations are uncorrelated for 30 lags and are t-distributed with 13.291 degrees of freedom. The process yields an expected annual return and annual volatility of approximately $8 \%$ and $16 \%$ respectively.

Table 1: Fitted parameter values, standard errors and $t$-statistics.

\begin{tabular}{c|c|c|c}
\hline \hline Parameter & Value & Standard Error & $t$-Statistic \\
\hline$K$ & $2.7084 \mathrm{e}-4$ & $1.1308 \mathrm{e}-004$ & 2.3952 \\
$\omega$ & $1.1744 \mathrm{e}-006$ & $2.0216 \mathrm{e}-7$ & 5.8096 \\
$\alpha_{1}$ & 0.0111 & 0.0065 & 1.7141 \\
$\beta_{1}$ & 0.9250 & 0.0068 & 135.5325 \\
$\psi_{1}$ & 0.1047 & 0.0108 & 9.6616 \\
DoF & 13.2910 & 1.7828 & 7.4551 \\
\hline
\end{tabular}

\section{A.2 Summary Statistics}

The summary statistics for the log-returns from the FTSE 100 index from $2^{\text {nd }}$ April 1984 to $15^{\text {th }}$ December 2010 inclusively is given in Table 3 . 
Table 2: Ljung-Box and Kolmogorov-Smirnov statistics at 5\% confidence interval.

\begin{tabular}{l|c|c}
\hline \hline & $\begin{array}{c}\text { Ljung-Box } \\
\text { (30 lags) }\end{array}$ & KS \\
\hline p-value & 0.5690 & 0.7568 \\
Critical value & 43.7730 & 0.0186 \\
Q-statistic & 28.0276 & - \\
KS Statistic & - & 0.0092 \\
\hline \hline
\end{tabular}

Table 3: Summary statistics for FTSE 100 daily log-returns.

\begin{tabular}{l|l||r|r}
\hline \hline & & Quantiles: & \\
Mean & $2.4741 \mathrm{e}-4$ & $1 \%$ & -0.0311 \\
Median & $6.0529 \mathrm{e}-4$ & $5 \%$ & -0.0168 \\
Std. dev. & 0.0112 & $10 \%$ & -0.0118 \\
Skewness & -0.3868 & $25 \%$ & -0.0054 \\
Kurtosis & 11.7876 & $75 \%$ & 0.0064 \\
Min & -0.1303 & $90 \%$ & 0.0118 \\
Max & 0.0938 & $95 \%$ & 0.0163 \\
& & $99 \%$ & 0.0286 \\
\hline \hline
\end{tabular}

